\title{
Dosimetric Comparison of IMRT Simultaneous Integrated Boost With Conventional IMRT Treatment Planning for Head and Neck Cancer
}

\author{
A. YAZICI ${ }^{a, *}$, B. DirICAN ${ }^{b}$, E. KARA $^{a}$ \\ ${ }^{a}$ Onko Ankara Oncology Center, Radiation Oncology Department, Ankara, Turkey \\ ${ }^{b}$ Gulhane Military Hospital, Radiation Oncology Department, Ankara, Turkey
}

\begin{abstract}
Intensity Modulated Radiation Therapy (IMRT) provides the ability to not only conform the radiation dose to the shape of the target thereby reducing dose to normal tissues, it also allows to deliver different doses each day to different parts of the target. This approach is known as a simultaneous integrated boost (SIB). This study aims that dosimetric parameters of SIB with IMRT treatment planning technique are compared with conventional treatment planning technique using NTCP for 2 head and neck cancer patients.
\end{abstract}

DOI: 10.12693/APhysPolA.128.B-236

PACS: 87.53.Tf, 87.55.D

\section{Introduction}

The simultaneous intefrated boost (SIB)-IMRT technique allows the simultaneous delivery of different dose levels to different target volumes within a single fraction [1].

Loco-regional failures remain a major concern following irradiation of locally advanced head and neck cancers; this has led radiation oncologists to investigate novel approaches that might offer better therapeutic indexes. It is now generally accepted that the modification of dose fractionation schedules can improve the therapeutic outcome by using accelerated or hyperfractionated regimes for fast growing tumours. Different strategies of hyperfractionation proved that an increase of $10-15 \%$ in the total dose is consistently associated with a $10-15 \%$ increase in local control rates without appreciable increase of late complications. Among acceleration, available data reveal that an acceleration of 1-2 weeks in the total treatment time, without total dose reduction, yielded approximately an increase of $15 \%$ over the control rate, still without worsening the late complication rates. In the concomitant boost schemes, a variation of the accelerated models, a second daily dose is delivered to a reduced target volume (at high risk of recurrence) during the course of conventionally fractionated radiotherapy.

A large phase III randomised clinical study was conducted by RTOG whose first report was published in 2000. Patients were assigned to four groups, standard fractionation, hyperfractionation, accelerated fractionation and accelerated fractionation with concomitant boost for the last 12 sessions. More than 1000 patients were analysed for outcome with a median follow-up

*corresponding author; e-mail: yaziciasli@yahoo.com of 23 months. Patients with hyperfractionation and accelerated fractionation with concomitant boost had significantly increased loco-regional control with respect to the conventional fractionation. All three altered fractionations increased significantly the acute complication rates, however no significant increase was observed for late effects. The recent clinical diffusion of intensitymodulated radiation therapy (IMRT) has added new interest in the field of concomitant boost irradiation. Given the promising superiority of IMRT techniques with respect to conventional and conformal techniques to deliver more conformal doses to targets sparing the organs at risk, it could then be justified to combine IMRT with dose escalation, until now hardly applicable to head and neck irradiation with conventional techniques due to excessive toxicity. Moreover the IMRT technique allows the planning and irradiation of different targets at different dose levels in a single treatment session, instead of two treatment plans in two daily sessions [2].

The aim of this study is to compare radiobiological effects between the SIB IMRT and conventional IMRT for treatment of head and neck patient using normal tissue complication probability (NTCP).

With the treatment of tumors, normal tissus receive a radiation dose, and the NTCP is the probability that a certain percentage of the patient population will incur unfavorable reactions in the contiguous tissue at a particular dose. We calculated NTCP of an organ for uniform dose $D$ to a volume $V$ using Lyman-KutcherBurman method, which is given by Eqs. (1-3):

$$
\mathrm{NTCP}=\frac{1}{2 \pi} \int_{-\infty}^{t} \mathrm{e}^{r^{2} / 2} \mathrm{~d} t
$$

where

$$
t=\frac{D-T D_{50}(V)}{m T D_{50}(V)} .
$$

The $T D_{50}(V)$ is the tolerance dose for a $50 \%$ probability of complication attributable to uniform irradiation of a 
partial volume $V$. That dose is related to the tolerance dose for the whole organ, $T D_{50}(1)$, by

$$
T D_{50}(V)=\frac{T D_{50}(1)}{V^{n}}
$$

In the foregoing equations, parameters $m$ and $n$ respectively deal with slope of the dose response curve and the volume effect.

\section{Methods and materials}

\subsection{Patient selection}

Two common head-and-neck cancer cases were chosen for treatment planning study. Patients were immobilized in the supine position with thermoplastic mask. Treatment planning CT data were acquired with a $3 \mathrm{~mm}$-slice thickness (Somatom Emotion Duo, Siemens, Germany). The target volumes and organ at risks were deliniated on the CT data set by the same radiation oncologist to prevent interphysician uncertainties. The target volumes considered were: including tumour bed, lymph nodes (cervical and supraclavicular) and tissues at risk for subclinical or microscopic disease and the OAR considered were: spinal cord, parotid glands, mandible and larynx.

\subsection{Planning techniques}

Two different radiation therapy techniques; 7 beams conventional inverse IMRT plans and SIB with IMRT plans were designed and optimized using the Prowess Panther DAQ treatment planning system (TPS) with $6 \mathrm{MV}$ photon beams from a Siemens Artiste equipped with Multileaf Collimator (MLC) with 160 leaves.

The beam geometry used seven non-coplanar radiation beams, gantry angles; $205^{\circ}, 255^{\circ}, 306^{\circ}, 0^{\circ}, 51^{\circ}, 102^{\circ}$, and $153^{\circ}$.

Inverse IMRT planning was carried out aiming firstly to optimise target volume coverage and secondly to spare organs at risk;

- target coverage: between 95 and $107 \%$ of the prescribed dose for each PTV,

- spinal cord: maximum significant dose of $45 \mathrm{~Gy}$, maximum point dose of $50 \mathrm{~Gy}$,

- parotid glands: 32 Gy to $2 / 3$ of the entire organ volume,

- larynx: $33 \%, 27 \%, 0 \%$ of the entire organ volume under the dose 50 Gy, 50 Gy, 66 Gy. Mean dose is under 44 Gy.

\subsection{Dose prescription}

First patient administered a total of 33 fractions, but with escalating doses per fraction (1.64, 1.73, and $2.18 \mathrm{~Gy}$ ) to achieve a total dose to the GTV of 54,57 , and 72 Gy and second patient total fraction number is 33 and doses per fraction (1.63, 1.82 and 2.12 Gy) to achieve a total dose to GTV of 70 Gy, respectively. The remaining target tissues were constrained to receive the same

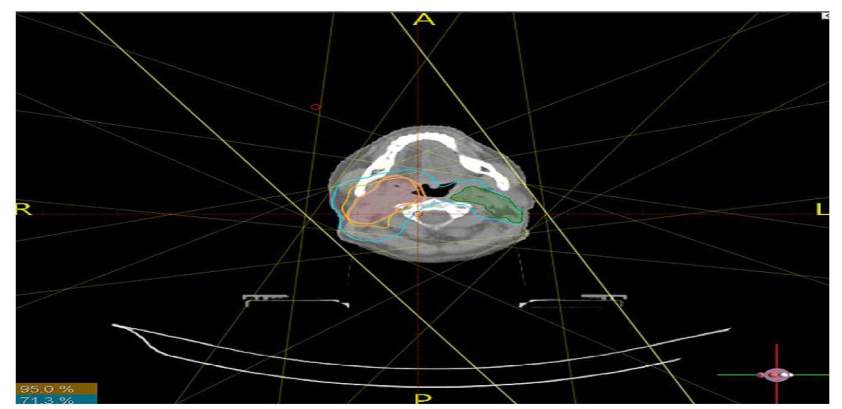

Fig. 1. The beam arrangement is shown on a CT slice where overlays show typical shape and relative positin of target volumes (PTV 54, PTV 72) and spinal cord.

dose in two patients regardless of the GTV dose level. The parotid glands were spared to the degree possible without compromising target coverage.

All these treatment plans were performed on the subsequently (SIB) and conventional (conv.) IMRT plans were compared using dosimetric parameters from dose-volume histograms (Fig. 2). NTCP for critical structures using doses of the organs at risk, and target volume coverage were analyzed. Results are given in Tables I and II.


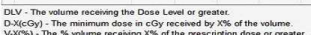

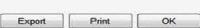

Fig. 2. Target and OaR dose results are shown on dose volume histogram DVH after simultaneous integrated IMRT plan for first patient.

\section{Conclusion}

From a radiobiological point of view, the SIB-IMRT appears to be an effective RT strategy for the primary treatment of $\mathrm{H} \& \mathrm{~N}$ cancers, and a new way to investigate RT acceleration. Based on available published studies on SIB-IMRT, the short term clinical outcome is very promising. However, very few data on late effects are available because of the short follow up time in the majority of reported studies.

The selected SIB fractionation schedule should always be compared in terms of radiobiological parameters with conventional fractionation regimes [1].

Doses of OaR with SIB-IMRT plans are found higher than conventional-IMRT plans but all critical doses are under the QUANTEC recommendations. 
TABLE I

DVH values using IMRT planning for first patient.

\begin{tabular}{|c|c|c|c|c|c|c|c|c|c|}
\hline $\mathrm{HN}$ & ptv72 & ptv57 & ptv54 & spinal cord & mandible & larynx & oral cavity & Parotid (L) & parotid $(\mathrm{R})$ \\
\hline \multicolumn{10}{|c|}{ SIB-IMRT planning for first patient } \\
\hline volume $\left[\mathrm{cm}^{3}\right]$ & 93.4 & 106 & 130.5 & 25.6 & 41.6 & 23.9 & 83.3 & 18.9 & 11.8 \\
\hline $\min .[\mathrm{cGy}]$ & 5894.5 & 4893.4 & 4506.1 & 859.2 & 1011.5 & 4719.8 & 1653.5 & 0 & 2082.4 \\
\hline $\max .[\mathrm{cGy}]$ & 7673.8 & 6746.9 & 5854.7 & 4646 & 7534.9 & 6496.7 & 6989.5 & 5277.4 & 7129.6 \\
\hline mean [cGy] & 7259.7 & 5807.1 & 5418.4 & 3546.1 & 3768.7 & 5407.2 & 3986.6 & 2408.4 & 4350.1 \\
\hline \multicolumn{10}{|c|}{ conventional IMRT planning for first patient } \\
\hline volume $\left[\mathrm{cm}^{3}\right]$ & 93.4 & 106 & 130.5 & 25.6 & 41.6 & 23.9 & 83.3 & 18.9 & 11.8 \\
\hline $\min$. [cGy] & 6176.9 & 3905.4 & 3246.3 & 707.8 & 960.3 & 3282.9 & 1192.8 & 0 & 2431.8 \\
\hline $\max .[\mathrm{cGy}]$ & 7629.6 & 7346.8 & 6156.3 & 4647.6 & 7331.3 & 5764.6 & 7010.6 & 5043.7 & 6955.9 \\
\hline mean [cGy] & 7339.5 & 5440.1 & 5201 & 2767.4 & 3665.4 & 4150.6 & 3536.4 & 2092 & 4298.1 \\
\hline \multicolumn{10}{|c|}{ SIB-IMRT planning for second patient } \\
\hline volume $\left[\mathrm{cm}^{3}\right]$ & 44.5 & 73.7 & 53.4 & 23.2 & - & 21.3 & 36.2 & 4.9 & 5.6 \\
\hline min. [cGy] & 6160.5 & 4961.3 & 4934.4 & 963.2 & - & 3781.1 & 2365.6 & 2531 & 1916.4 \\
\hline $\max .[\mathrm{cGy}]$ & 7271.9 & 7199.9 & 5694.7 & 4433.2 & - & 7014.8 & 6155.8 & 6158.7 & 4649.1 \\
\hline mean $[\mathrm{cGy}]$ & 6917.2 & 6115.5 & 5337.9 & 3566.4 & - & 5119 & 4716.2 & 4265.5 & 2857.6 \\
\hline \multicolumn{10}{|c|}{ conventional IMRT planning for second patient } \\
\hline volume $\left[\mathrm{cm}^{3}\right]$ & 44.5 & 73.7 & 53.4 & 23.2 & - & 21.3 & 36.2 & 4.9 & 5.6 \\
\hline min. [cGy] & 6316.7 & 5139.6 & 4427.6 & 432.7 & - & 2863.1 & 1570.7 & 3478.2 & 1683.6 \\
\hline $\max .[\mathrm{cGy}]$ & 7375.2 & 7375.2 & 6245.8 & 4598.5 & - & 6739.7 & 5848.4 & 6875 & 4175.2 \\
\hline mean [cGy] & 7151.4 & 6335.8 & 5324.6 & 2982.9 & - & 4344.5 & 4035.4 & 5211 & 2522 \\
\hline
\end{tabular}

TABLE II

NTCP values for critical structures.

\begin{tabular}{c|c|c|c|c|c|c}
\hline \hline NTCP & spinal cord & larynx & mandibula & oral cavity & parotid $(\mathrm{L})$ & parotid (R) \\
\hline H2 - SIB & 0.0094 & 0.0081 & - & 0.0097 & 0.3968 & 0.0279 \\
H2 - conv. & 0.0079 & 0.0070 & - & 0.0083 & 0.8061 & 0.0110 \\
H1 - SIB & 0.0093 & 0.0084 & 0.0074 & 0.0084 & 0.0046 & 0.4633 \\
H1 - conv. & 0.0074 & 0.0066 & 0.0072 & 0.0076 & 0.0088 & 0.4348
\end{tabular}

With respect to NTCP there is no significant differences between SIB IMRT planning and conventional IMRT planning.

For the spinal cord, larynx, mandible and oral cavity the NTCP values were calculated to be smaller than $1 \%$ for both patients. The maximum NTCP was 0.8061 for parotid glands (L) of second patient because of overlapping with tumour.

SIB-IMRT is more efficient and more accurate on tumour covarage than conventional-IMRT.

The SIB approach provides the ability to deliver higher than conventional doses safely to the tumor and involved lymph nodes while reducing the overall treatment course. Patients treated in this fashion are able to complete treatment faster than patients treated with conventional techniques. Not only is treatment delivered faster, it is more effective than standard approaches.

\section{References}

[1] E. Orlandi, M. Pallazzi, E. Pignoli, C. Fallai, A. Giostra, P. Olmi, Crc. Cr. Rev. Oncol.-Hem. 73, 111 (2010).

[2] A. Fogliata, A. Bolsi, L. Cozzi, J. Bernier, Radiother. Oncol. 69, 267 (2003). 